\title{
Angular and polarization trails from effective interactions of Majorana neutrinos at the $\mathrm{LHeC}$
}

\author{
Lucía Duarte $^{1, \mathrm{a}} \mathbb{\mathbb { O }}$, Gabriel Zapata ${ }^{2}$, Oscar A. Sampayo ${ }^{2, \mathrm{~b}}$ \\ ${ }^{1}$ Instituto de Física, Facultad de Ingeniería, Universidad de la República, Julio Herrera y Reissig 565, 11300 Montevideo, Uruguay \\ ${ }^{2}$ Departamento de Física, Universidad Nacional de Mar del Plata, Instituto de Investigaciones Físicas de Mar del Plata (IFIMAR), CONICET, \\ UNMDP, Funes 3350, 7600 Mar del Plata, Argentina
}

Received: 22 February 2018 / Accepted: 21 April 2018 / Published online: 30 April 2018

(C) The Author(s) 2018

\begin{abstract}
We study the possibility of the LHeC facility to disentangle different new physics contributions to the production of heavy sterile Majorana neutrinos in the lepton number violating channel $e^{-} p \rightarrow l_{j}^{+}+3$ jets $\left(l_{j} \equiv e, \mu\right)$. This is done investigating the angular and polarization trails of effective operators with distinct Dirac-Lorentz structure contributing to the Majorana neutrino production, which parameterize new physics from a higher energy scale. We study an asymmetry in the angular distribution of the final anti-lepton and the initial electron polarization effect on the number of signal events produced by the vectorial and scalar effective interactions, finding both analyses could well separate their contributions.
\end{abstract}

\section{Introduction}

The discovery of neutrino masses through oscillation experiments continues to be the most compelling evidence of physics beyond the standard model (SM). And yet the seesaw mechanism for neutrino mass generation [1-6] plays a role as the most straightforward means to explain the tiny neutrino mass values. This mechanism introduces right-handed sterile neutrinos $N_{i}$ which can have a Majorana mass term leading to the tiny known masses for the standard neutrinos, as long as the Yukawa couplings between the right-handed Majorana neutrinos and the standard ones remain small. In fact, for Yukawa couplings of order $Y \sim 1$, a Majorana mass scale of order $M_{N} \sim 10^{15} \mathrm{GeV}$ is needed to account for a light neutrino mass compatible with the current neutrino data $\left(m_{v} \sim 0.01 \mathrm{eV}\right)[7]$. On the other hand, for smaller Yukawa couplings, of order $Y \sim 10^{-8}-10^{-6}$, sterile neutrinos with masses around $M_{N} \sim(1-1000) \mathrm{GeV}$ could exist,

\footnotetext{
a e-mail: 1duarte@ fing.edu.uy

be-mail: sampayo@mdp.edu.ar
}

but in the simplest Type-I seesaw model with sterile Majorana neutrinos, this leads to negligible neutrino mixing values $U_{l N}^{2} \sim m_{v} / M_{N} \sim 10^{-14}-10^{-10}[8,9]$. Thus, both alternatives lead to the decoupling of the Majorana neutrinos. In this scenario, the observation of lepton number violating (LNV) processes allowed by the existence of a Majorana neutrino mass term would be a sign of physics beyond the minimal seesaw mechanism [10].

From the theoretical point of view, an alternative approach is to consider the Majorana neutrino interactions as originating in physics from a higher energy scale, parameterized by a model independent effective Lagrangian $[10,11]$. We consider that the sterile $N$ interacts with the SM particles by higher dimension effective operators, and take these interactions to be dominant in comparison with the mixing with light neutrinos through the Yukawa couplings, which we neglect. In this sense we depart from the usual viewpoint in which the sterile neutrinos mixing with the standard neutrinos is assumed to govern the $N$ production and decay mechanisms $[9,12]$.

The possibility for lepton number violation evidencing the Majorana nature of neutrinos in past and future hadron and lepton colliders has been extensively studied in the context of seesaw models (see $[8,13]$ and the references therein). The two-unit LNV channel $e^{-} p \rightarrow l_{j}^{+}+3$ jets has been studied in electron-proton colliders in [14-18]. In this context, the LHeC proposed collider $[19,20]$ offers an opportunity to test the sterile neutrino interactions in this channel in a clean environment, as well as other interesting new physics models [21-24].

The effective interactions we consider here for the heavy Majorana neutrinos were early studied in [10], where the possible phenomenology of dimension- 6 effective operators was introduced. The dimension-5 operators extending the low-scale Type-I seesaw were investigated in [25]. Their phenomenology is addressed in recent work [26,27]. Dimension 
7 effective $N$ operators are studied in [28,29]. The collider phenomenology of the dimension-6 effective Lagrangian used in this paper has been studied by our group and others in [10,30-36].

The different operators in the effective Lagrangian parameterize a wide variety of UV-complete new physics models, like extended scalar and gauge sectors as the left-right symmetric model, vector and scalar leptoquarks, etc. Thus, discerning between the possible contributions given by them to specific processes gives us a hint on what kind of new physics at a higher energy regime is responsible for the observed interactions.

In [32] we studied the potential of the $\mathrm{LHeC}$ to discover Majorana neutrinos for different values of their mass, effective couplings and the new physics scale. Here we aim to go further in the study of the Majorana neutrino effective interactions, and point towards disentangling the possible contributions of effective operators with different Dirac-Lorentz structure to the $e^{-} p \rightarrow l^{+}+3$ jets process with the aid of angular distributions and polarization effects.

We study the influence of vectorial and scalar operators on the angular distribution of the final anti-lepton, building a forward-backward asymmetry, and study the potential of using the initial electron polarization as a discriminator between both effective operator groups.

The paper is organized as follows. In Sect. 2 we review the effective Lagrangian and the existing constraints on the values of the effective couplings. In Sect. 3 we show the analytic and numerical signal calculation, as well as the cuts imposed for background suppression. The numerical results for the final anti-lepton angular distributions and forwardbackward asymmetry are presented in Sect. 4, and the initial electron polarization analysis is presented in Sect. 5. Our conclusions are drawn in Sect. 6.

\section{Majorana neutrino interaction model}

\subsection{Effective operators and Lagrangian}

As the heavy sterile Majorana neutrino $N$ is a SM singlet, its only possible renormalizable interactions with SM fields involve the Yukawa couplings, which must be very small in order to accommodate the observed tiny ordinary $v$ masses. Thus, any observation of leptonic number non-conservation should be a manifestation of physics beyond the minimal seesaw mechanism [10]. Our aim is to investigate the possible contributions of a heavy Majorana neutrino with negligible mixing to the $\mathrm{SM} v_{L}$. We consider the most simple scenario, including only one heavy neutrino state $N$ as an observable degree of freedom.

The effects of the new physics involving one heavy sterile neutrino and the SM fields are parameterized by a set of effective operators $\mathcal{O}_{\mathcal{J}}$ satisfying the $S U(2)_{L} \otimes U(1)_{Y}$ gauge symmetry [37]. The contribution of these operators to observable quantities is suppressed by inverse powers of the new physics scale $\Lambda$. The total Lagrangian is organized as follows:

$\mathcal{L}=\mathcal{L}_{S M}+\sum_{n=5}^{\infty} \frac{1}{\Lambda^{n-4}} \sum_{\mathcal{J}} \alpha_{\mathcal{J}} \mathcal{O}_{\mathcal{J}}^{(n)}$

where $n$ is the mass dimension of the operator $\mathcal{O}_{\mathcal{J}}^{(n)}$.

Note that we do not include the Type-I seesaw Lagrangian - the Majorana and Yukawa terms - giving rise to the mixing between the sterile and the standard left-handed neutrinos, which we are neglecting. In this work it is considered that the dominating new physics effects leading to the lepton number violation come from the lower dimension operators that can be generated at tree level in the unknown underlying renormalizable theory.

The dimension-5 operators in (1) were studied in detail in [25]. These include the well-known Weinberg operator $\mathcal{O}_{W} \sim(\bar{L} \tilde{\phi})\left(\phi^{\dagger} L^{c}\right)$ [38] contributing to the light neutrino masses, and operators with the $N: \mathcal{O}_{N \phi} \sim\left(\bar{N} N^{c}\right)\left(\phi^{\dagger} \phi\right)$ contributing to the $N$ Majorana masses and giving couplings of the heavy neutrinos to the Higgs (its phenomenology for the LHC has been studied very recently in [27]), and an operator $\mathcal{O}_{N B}^{(5)} \sim\left(\bar{N} \sigma_{\mu \nu} N^{c}\right) B^{\mu \nu}$ inducing magnetic moments for the heavy neutrinos, which is identically zero if we include just one sterile neutrino $N$ in the theory. ${ }^{1}$ In the following, as the dimension-5 operators do not contribute to the studied processes - discarding the heavy-light neutrino mixings we will only consider the contributions of the dimension- 6 operators, following the treatment made in [10].

We organize the effective operators in different subsets. The first one includes operators with scalar and vector bosons (SVB),

$$
\begin{aligned}
& \mathcal{O}_{L N \phi}=\left(\phi^{\dagger} \phi\right)(\bar{L} N \tilde{\phi}), \quad \mathcal{O}_{N N \phi}=i\left(\phi^{\dagger} D_{\mu} \phi\right)\left(\bar{N} \gamma^{\mu} N\right) \\
& \mathcal{O}_{N e \phi}=i\left(\phi^{T} \in D_{\mu} \phi\right)\left(\bar{N} \gamma^{\mu} l\right)
\end{aligned}
$$

and a second subset includes the baryon-number conserving 4-fermion contact terms:

$$
\begin{aligned}
& \mathcal{O}_{d u N e}=\left(\bar{d} \gamma^{\mu} u\right)\left(\bar{N} \gamma_{\mu} l\right), \quad \mathcal{O}_{f N N}=\left(\bar{f} \gamma^{\mu} f\right)\left(\bar{N} \gamma_{\mu} N\right), \\
& \mathcal{O}_{L N L e}=(\bar{L} N) \epsilon(\bar{L} l), \mathcal{O}_{L N Q d}=(\bar{L} N) \epsilon(\bar{Q} d), \\
& \mathcal{O}_{Q u N L}=(\bar{Q} u)(\bar{N} L), \quad \mathcal{O}_{Q N L d}=(\bar{Q} N) \epsilon(\bar{L} d)
\end{aligned}
$$

where $e_{i}, u_{i}, d_{i}$ and $L_{i}, Q_{i}$ denote, for the family labeled $i$, the right-handed $S U(2)$ singlet and the left-handed $S U(2)$ doublets, respectively. Here $\gamma^{\mu}$ are the Dirac matrices, and $\epsilon=i \sigma^{2}$ is the antisymmetric symbol. We do not consider

\footnotetext{
${ }^{1}$ The effects of considering the $\mathcal{O}_{N B}^{(5)}$ operator were studied in [25] for the case of 2 massive Majorana neutrinos $N_{1,2}$. Our treatment coincides with the limit in which $N_{1,2}$ are mass-degenerate and the light-heavy mixing is taken to be zero.
} 


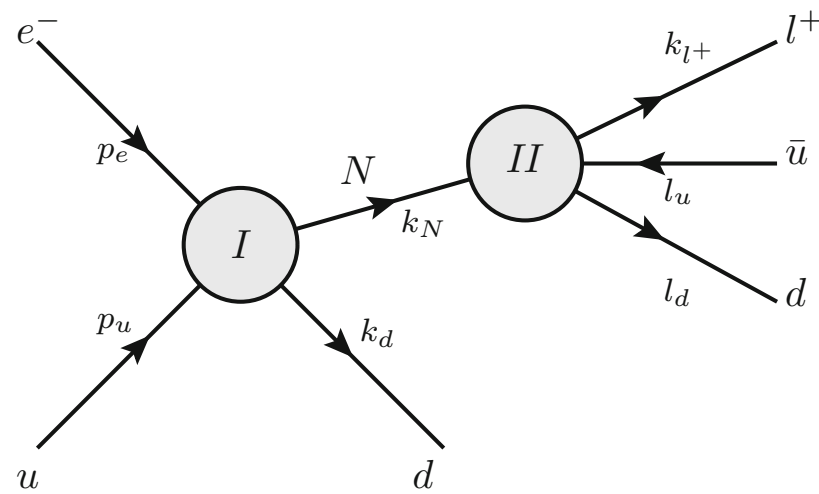

Fig. 1 Process ep $\rightarrow l^{+}+3$ jets $+X$ with $\mathrm{N}$ decaying according to Refs. $[33,34]$

the one-loop generated operators which are naturally suppressed by a factor $1 / 16 \pi^{2}[10,39]$. The complete expression for the dimension- 6 effective Lagrangian can be found in an appendix in [34].

The effective operators in (2) and (3) cover a wide variety of new physics models, as we mentioned in the introduction. The effects of the four-fermion contact operators $\mathcal{O}_{d u N e} \equiv \mathcal{O}_{V_{0}}$ and $\mathcal{O}_{Q N L d} \equiv \mathcal{O}_{S_{3}}$ have been studied recently as a parameterization of the minimal left-right Symmetric Model (LRSM) in recasts of LHC searches for the same-sign dilepton signal [40].

In order to obtain the interactions involved in the process ep $\rightarrow \mathrm{l}^{+}+3$ jets depicted in Fig. 1 we consider the effective Lagrangian terms involved in the calculations, taking the scalar doublet after spontaneous symmetry breaking as $\phi=\left(\begin{array}{c}0 \\ \frac{v+h}{\sqrt{2}}\end{array}\right)$, with $h$ being the Higgs field and $v$ its v.e.v. For the Majorana neutrinos production $(I)$ and decay (II) vertices in Fig. 1 we have contributions to the effective Lagrangian related to the spontaneous symmetry breaking process coming from (2) and the four-fermion interactions involving quarks and leptons from (3):

$$
\begin{aligned}
\mathcal{L}_{e f f}= & \frac{1}{\Lambda^{2}}\left\{-\frac{m_{W} v}{\sqrt{2}} \alpha_{W}^{(i)} W^{\dagger \mu} \bar{N}_{R} \gamma_{\mu} e_{R, i}\right. \\
& +\alpha_{V_{0}}^{(i, j)} \bar{d}_{R, i} \gamma^{\mu} u_{R, i} \bar{N}_{R} \gamma_{\mu} e_{R, j} \\
& +\alpha_{S_{1}}^{(i, j)}\left(\bar{u}_{L, i} u_{R, i} \bar{N} v_{L, j}+\bar{d}_{L, i} u_{R, i} \bar{N} e_{L, j}\right) \\
& +\alpha_{S_{2}}^{(i, j)}\left(\bar{v}_{L, i} N_{R} \bar{d}_{L, i} d_{R, j}-\bar{e}_{L, i} N_{R} \bar{u}_{L, i} d_{R, j}\right) \\
& \left.+\alpha_{S_{3}}^{(i, j)}\left(\bar{u}_{L, i} N_{R} \bar{e}_{L, i} d_{R, j}-\bar{d}_{L, i} N_{R} \bar{v}_{L, i} d_{R, j}\right)+\text { h.c. }\right\}
\end{aligned}
$$

where the sum over the fermion families $i, j=1,2,3$ is understood and the couplings $\alpha_{\mathcal{J}}^{(i, j)}$ are associated to specific operators according to
$\alpha_{W}=\alpha_{N e \phi}, \alpha_{V_{0}}=\alpha_{d u N e}, \alpha_{S_{1}}=\alpha_{Q u N L}, \alpha_{S_{2}}=\alpha_{L N Q d}$,

$\alpha_{S_{3}}=\alpha_{Q N L d}$.

In this work we allow for family mixing in the interactions involving two or more different SM leptons: this allows for the appearance of $\mu^{+}$anti-leptons together with positrons in the final state. The case $l^{+}=\tau^{+}$is allowed in theory, but we do not take it into account, due to the difficult tau reconstruction in experiments.

The effective operators above can be classified by their Dirac-Lorentz structure into scalar and vectorial. The scalar and vectorial operators contributing to the studied processes are those appearing in (4) with couplings named $\alpha_{S_{1,2,3}}$ and $\alpha_{W, V_{0}}$, respectively.

The relative sizes between the different effective couplings are given by the contribution of the corresponding operators to the experimental observables.

\subsection{Effective coupling bounds summary}

The current experimental constraints on the light-heavy neutrino mixing parameters in seesaw models can be exploited to impose plausible constraints on the numerical values of the effective couplings $\alpha_{\mathcal{J}}$, which weight the relative importance of the possible effective interactions.

In the literature [13,41-44] the existing experimental bounds are summarized in general phenomenological approaches considering low-scale minimal seesaw models, parameterized by a single heavy neutrino mass scale $M_{N}$ and a light-heavy mixing $U_{l N}$, with $l$ indicating the lepton flavor. We exploit these results - like constraints on processes as neutrino-less double beta decay $(0 \nu \beta \beta)$, electroweak precision data (EWPD), LNV rare meson decays as well as direct collider searches, including $Z$ decays - linking the $U_{l N}$ mixings in Type-I seesaw models $[9,12]$ with our effective couplings in (1) by the ad hoc relation

$U_{l N}^{2} \simeq\left(\frac{\alpha v^{2}}{2 \Lambda^{2}}\right)^{2}$.

In previous work $[33,34]$ we have presented our procedure, and refer the reader to those papers for a detailed discussion.

For the couplings involving the first fermion family - taking indices $i=1$ and $j=1$ in (4) - the most stringent are the $0 v \beta \beta$-decay bounds obtained by the KamLAND-Zen collaboration [45]. Following the treatment made in [34,41,46], they give us an upper limit $\alpha_{0 \nu \beta \beta}^{\text {bound }} \leq 3.2 \times 10^{-2}\left(\frac{m_{N}}{100 \mathrm{GeV}}\right)^{1 / 2}$, where the new physics scale is taken to be $\Lambda=1 \mathrm{TeV}$ (here and in the following). ${ }^{2}$ For the second fermion family - taking indices $i=2$ or $j=2$ in (4) - and sterile neutrino

\footnotetext{
$\overline{2}$ The new physics scale $\Lambda=1 \mathrm{TeV}$ is taken as an illustration. One can obtain the values at any other scale $\Lambda^{\prime}$ considering $\alpha_{\mathcal{J}}^{\prime}=\left(\frac{\Lambda^{\prime}}{\Lambda}\right)^{2} \alpha_{\mathcal{J}}$.
} 
Table 1 Effective couplings numerical values

$\alpha_{0 \nu \beta \beta}^{\text {bound }}=3.2 \times 10^{-2}\left(\frac{m_{N}}{100 \mathrm{GeV}}\right)^{1 / 2}, \quad \alpha_{E W P D}^{\text {bound }}=0.32$

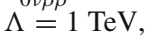

$\alpha_{W}^{(1)}=\alpha_{0 \nu \beta \beta}^{\text {bound }}$

$\alpha_{W}^{(2)}=\alpha_{E W P D}^{\text {bound }}$

$\alpha_{V_{0}}^{(1,1)}=\alpha_{0 \nu \beta \beta}^{\text {bound }}$

$\alpha_{V_{0}}^{(1,2)}=\alpha_{V_{0}}^{(2,1)}=\alpha_{V_{0}}^{(2,2)}$

$=\alpha_{E W P D}^{\text {bound }}$

$\alpha_{S_{1,2,3}}^{(1,1)}=\alpha_{0 \nu \beta \beta}^{\text {bound }}$

$\alpha_{S_{1,2,3}}^{(1,2)}=\alpha_{S_{1,2,3}}^{(2,1)}=\alpha_{S_{1,2,3}}^{(2,2)}$ $=\alpha_{E W P D}^{\text {bound }}$

masses in the range $m_{W} \lesssim m_{N}$ the upper limits come from EWPD like radiative lepton flavor violating (LFV) decays as $\mu \rightarrow$ e $\gamma[41,44,47,48]$ giving a bound $\alpha_{E W P D}^{\text {bound }} \leq 0.32$.

In our numerical analysis throughout the paper - for the sake of simplicity - we take the couplings associated to the operators that contribute to the $0 \nu \beta \beta$-decay for the first family $(i, j=1)$ as restricted by the corresponding bound $\alpha_{0 \nu \beta \beta}^{\text {bound }}$, and we fix the other couplings to the value $\alpha^{\text {bound }} \leq 0.32$ valid for high Majorana neutrino masses, ${ }^{3}$ as detailed in Table 1.

\section{Signal detection}

The $\mathrm{LHeC}$ is proposed to be an $e^{-} p$ collider built at the LHC tunnel, using an electron beam in the $60-150 \mathrm{GeV}$ energy range with the existing $7 \mathrm{TeV}$ proton beam. It is expected to achieve an integrated luminosity $\mathcal{L}=100 \mathrm{fb}^{-1}$ per operation year. One possibly very crucial feature of the LHeC is the availability of a polarized electron beam [19,20], which has already been studied as a chance to enhance the observability of Majorana neutrinos in the context of the left-right symmetric SM extension [22,24].

In this paper we study the effects of the possible existence of a heavy sterile Majorana neutrino $N$ with effective interactions on the angular distribution of the produced antilepton and the effects of the initial electron polarization in the process $e p \rightarrow l_{j}^{+}+3$ jets $\left(l_{j} \equiv e, \mu\right)$ at the LHeC.

The cross section for the process ep $\rightarrow l^{+}+3$ jets is calculated using the Lagrangian in (4), according to the process in Fig. 1. The analytical expression is

$\sigma\left(e p \rightarrow l^{+}+3 \mathrm{jets}\right)=\sum_{i} \int_{m_{N}^{2} / s}^{1} \mathrm{~d} x f_{i}(x) \hat{\sigma}_{i}(x s)$

where the center-of-mass energy is taken to be $\sqrt{s}=$ $\sqrt{4 E_{e} E_{p}}, \hat{\sigma}$ is the parton level scattering cross section and $\hat{s}$ the squared center-of-mass energy. Here $i=1$ corresponds to the channel $e u \rightarrow N d$ and $i=2$ to the crossed channel

\footnotetext{
3 Allowing for family mixing does not impose severe bounds in the high $m_{N}$ range we are considering in this paper.
}

$e \bar{d} \rightarrow N \bar{u}$. The function $f_{1}(x)$ represents the $u(x)$ parton distribution function (PDF), and $f_{2}(x)$ the one for $\bar{d}(x)$. For numerical calculations we use the CTEQ set [49].

The parton level cross section is written as

$\hat{\sigma}_{i}(x s)=\int(2 \pi)^{4} \delta^{(4)}\left(p_{e}+p_{u}-\sum_{j=1,4} k_{j}\right) \overline{\left|M_{(i)}\right|^{2}} \prod_{j=1,4} \frac{d^{4} k_{j}}{2 \pi^{3}}$,

and the squared scattering amplitudes in the narrow width approximation are :

$$
\begin{aligned}
{\overline{\mid M_{(i)}}}^{2}= & \left(\frac{\pi}{4 m_{N} \Gamma_{N} \hat{s}}\right) \delta\left(k_{N}^{2}-m_{N}^{2}\right)\left|\Lambda_{(I), i}\right|^{2}\left(\left|\Lambda_{(I I)}^{(+)}\right|^{2}\right. \\
& \left.+\left|\Lambda_{I I}^{(-)}\right|^{2}\right)
\end{aligned}
$$

where $^{4}$

$$
\begin{aligned}
\left|\Lambda_{(I), 1}\right|^{2}= & \frac{4}{\Lambda^{2}}\left[4\left(\alpha_{S_{2}}\left(\alpha_{S_{2}}-\alpha_{S_{3}}\right)+\alpha_{S_{1}}^{2}\right)\left(k_{d} \cdot p_{u}\right)\left(k_{N} \cdot p_{e}\right)\right. \\
& +\left(4 \alpha_{W}^{2}\left|\Pi_{W}^{(2)}\right|^{2}+\alpha_{S_{3}}\left(\alpha_{S_{3}}-\alpha_{S_{2}}\right)\right)\left(k_{d} \cdot p_{e}\right)\left(k_{N} \cdot p_{u}\right) \\
& \left.+\left(\alpha_{S_{3}} \alpha_{S_{2}}+4 \alpha_{V_{0}}^{2}\right)\left(k_{d} \cdot k_{N}\right)\left(p_{e} \cdot p_{u}\right)\right] \\
\left|\Lambda_{(I I)}^{(-)}\right|^{2}= & \frac{16}{\Lambda^{4}}\left[\left|\Pi_{W}^{(2)}\right|^{2} \alpha_{W}^{2}\left(k_{N} \cdot l_{u}\right)\left(k_{l^{+}} \cdot l_{d}\right)\right. \\
& \left.+\alpha_{V_{0}}^{2}\left(k_{N} \cdot l_{d}\right)\left(k_{l^{+}} \cdot l_{u}\right)\right] \\
\left|\Lambda_{(I I)}^{(+)}\right|^{2}= & \frac{4}{\Lambda^{4}}\left[\left(\alpha_{S_{1}}^{2}+\alpha_{S_{2}}^{2}-\alpha_{S_{2}} \alpha_{S_{3}}\right)\left(l_{u} \cdot l_{d}\right)\left(k_{l^{+}} \cdot k_{N}\right)\right. \\
& +\left(\alpha_{S_{3}}^{2}-\alpha_{S_{2}} \alpha_{S_{3}}\right)\left(k_{l^{+}} \cdot l_{d}\right)\left(l_{u} \cdot k_{N}\right) \\
& \left.+\alpha_{S_{2}} \alpha_{S_{3}}\left(l_{u} \cdot k_{l^{+}}\right)\left(l_{d} \cdot k_{N}\right)\right]
\end{aligned}
$$

with $\Pi_{W}^{(1)}=m_{W}^{2} /\left(-2\left(p_{u} \cdot k_{d}\right)-m_{W}^{2}\right), \Pi_{W}^{(2)}=m_{W}^{2} /\left(2\left(l_{u}\right.\right.$. $\left.\left.l_{d}\right)-m_{W}^{2}\right)$. The final leptons can be either $e^{+}$or $\mu^{+}$since this is allowed by the interaction Lagrangian (4). These final states are clear signals for intermediary Majorana neutrinos, thus we sum the cross section over the flavors of the final leptons. The total width $\left(\Gamma_{N}\right)$ for the Majorana neutrino decay is calculated in [34].

The numerical cross section for the Majorana neutrino production in $e p$ colliders and the following decay $N \rightarrow$ $l^{+}+2$ jets is updated from [32], considering the values for the effective couplings in Table 1 , and the full $N$ decay width calculated in [34].

In Fig. 2a we show the results for the cross section, as a function of the Majorana neutrino mass $m_{N}$, for the electron beam energy $E_{e}=150 \mathrm{GeV}$ and for a $E_{P}=7 \mathrm{TeV}$ proton beam. We have considered $\sqrt{\hat{s}}<\Lambda$ in order to ensure the validity of the effective Lagrangian approach. The effective couplings are taken as in Table 1 . The phase space integration of the squared amplitude is made generating the final momenta with the Monte Carlo routine RAMBO [50].

\footnotetext{
${ }^{4}$ Here and in Sect. 5 we omit the family superscripts in the effective couplings for simplicity.
} 


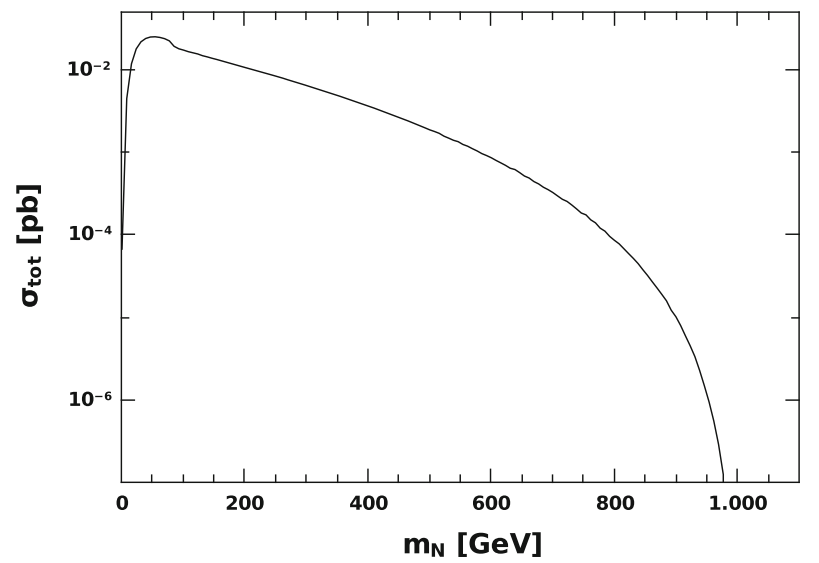

(a) Signal cross section. Effective numerical values

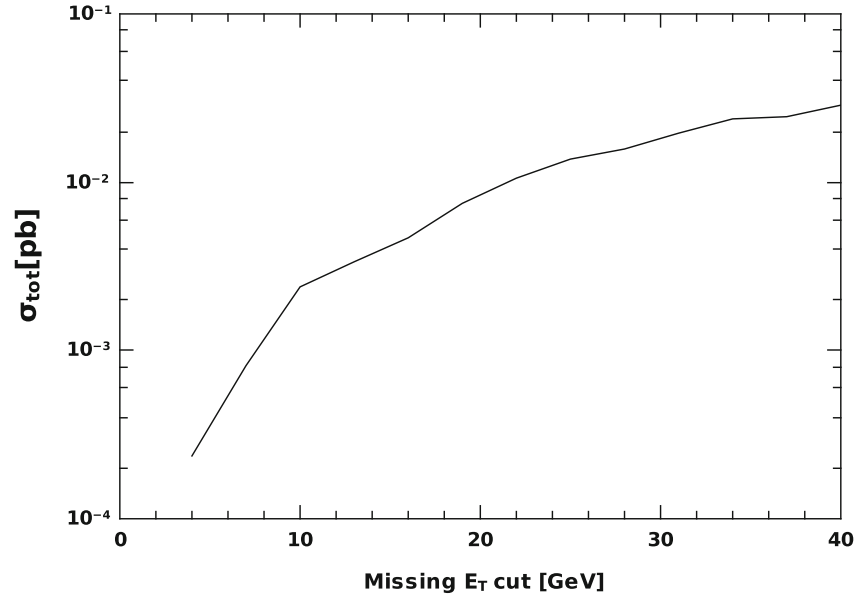

(b) Background $E_{T}$ dependence.

Fig. 2 Cross section for the process $e p \rightarrow l^{+}+3$ jets with $\mathrm{N}$ decaying according to Refs. [33,34] (a) and background dependence with missing $E_{T}(\mathbf{b})$

Although the lepton number violating considered signal is strictly forbidden in the SM, the backgrounds for the studied process were carefully investigated in $[15,32]$. The dominant background comes from $W \rightarrow l^{+}\left(e^{+}, \mu^{+}\right)$events. The process $e^{-} p \rightarrow e^{-} l^{+} j j j v$ is not distinguished from the signal if the outgoing electron is lost in the beamline, and is dominated by the exchange of an almost real photon with a very collinear outgoing electron $\left(p \gamma \rightarrow l^{+}+3 j+v\right)$, which convoluted with the PDF representing the probability of finding a photon inside an electron, is found to be the major contribution to $W$ production. In this paper we have updated the simulation of background processes, done with CalcHep [51].

As the SM background always involves final state neutrinos, in $[15,32]$ was found that a cut on the missing $E_{T}$ helps in reducing this background. Other efficient cut is on the $l^{+}$minimum transverse momentum. In Fig. 2b we show the behavior of the background with the maximum missing energy $E_{T}$ for $E_{e}=150 \mathrm{GeV}$. A cut of $E_{T, \max } \leq 10 \mathrm{GeV}$, which is a reasonable value for the detector resolution, has not appreciable effects on the signal but reduces the background significantly. In Fig. 3 we show the differential cross section for the background and the signal for different values of the Majorana masses as a function of the transverse momentum $p_{T, l^{+}}$of the anti-lepton. In this figures the cut on the missing energy $E_{T}$ has already been included. It can be seen that the background is mostly concentrated at low values of $p_{T, l^{+}}$, and a cut imposed on $p_{T, l^{+}}^{\min }$ could be effective to improve the signal/background relation.

Finally, in Fig. 4 we show a plot comparing the magnitude of the signal for different values of the Majorana neutrino mass, and the background for different $E_{T \text {, max }}$ cuts (black dashed lines), depending on the $p_{T, l^{+}}^{\min }$ cut imposed. In the figure the arrows indicate the value of the cuts used in the

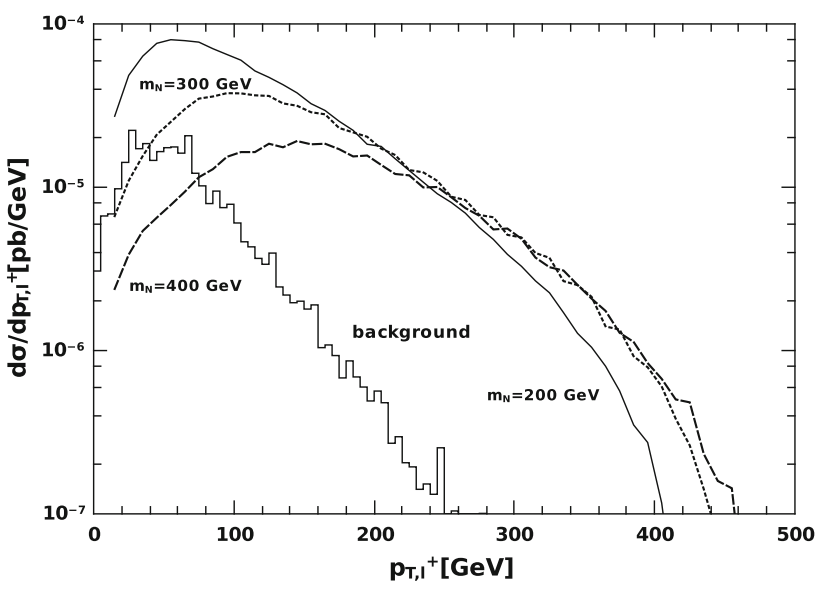

Fig. 3 Differential cross section of signal and background in function of transverse momentum $p_{T, l^{+}}$. The cut in missing $E_{T}$ is included

analysis: we impose $p_{T, l^{+}} \geq 90 \mathrm{GeV}$ and $E_{T \text {,miss }} \leq 10$ $\mathrm{GeV}$ in order to reduce the background without appreciably decreasing the signal.

\section{Angular distributions and asymmetry}

The use of angular asymmetries to disentangle the contributions from effective operators with different Dirac-Lorentz structure was already proposed in [10]. A forward-backward like asymmetry was studied in [35] for the case of long-lived Majorana neutrinos in the well-known dilepton LNV channel at the LHC. Recently, a forward-backward asymmetry is used to disentangle the Dirac or Majorana nature of intermediate neutrinos in purely leptonic $N$ decays at the LHC [52]. 


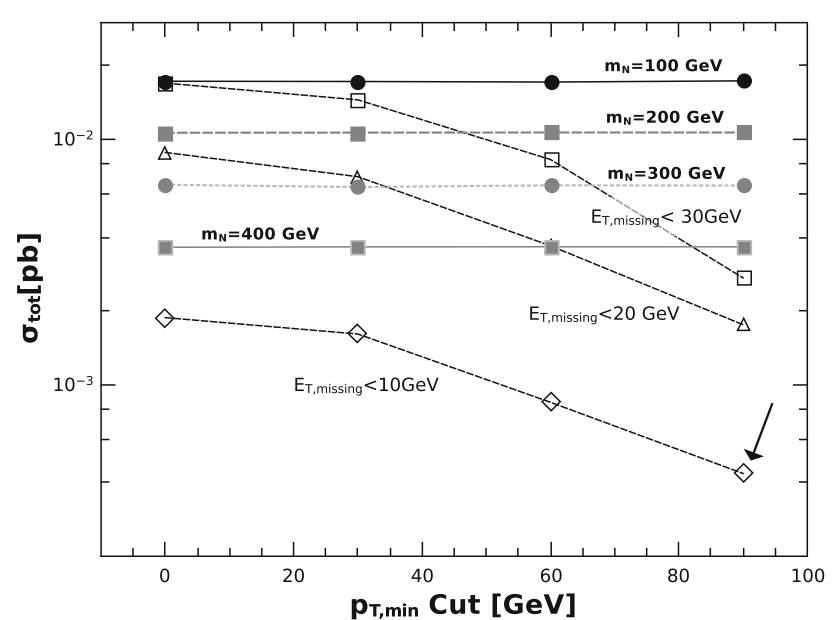

Fig. 4 Comparison between signal and background for different Majorana neutrino masses, cut in missing $E_{T}$ and the transversal momentum of the final lepton $p_{T, l^{+}}$. The arrows indicate the cuts and backgrounds used in the analysis

With the final anti-lepton angular distribution $d \sigma / d \cos \theta$ where $\theta$ is the angle between the outgoing anti-lepton and the incident electron beam in the lab frame, we construct a forward-backward asymmetry $A_{F B}^{l^{+}}$as a function of the number of events in each hemisphere:

$A_{\mathrm{FB}}^{l_{+}^{+}}=\frac{N_{+}-N_{-}}{N_{+}+N_{-}}$,

where $N_{+}$is the number of events with an angle in the interval $0 \leq \theta \leq \pi / 2$ and $N_{-}$the number of events with $\pi / 2 \leq \theta \leq$ $\pi$.

To measure the effects from the scalar operators we set the effective couplings corresponding to the vectorial operators $\alpha_{W}^{(i)}$ and $\alpha_{V_{0}}^{(i, j)}$ equal to zero, and set the value of the scalar couplings $\alpha_{S_{1,2,3}}^{(i, j)}$ in (10) to the values in Table 1 corresponding

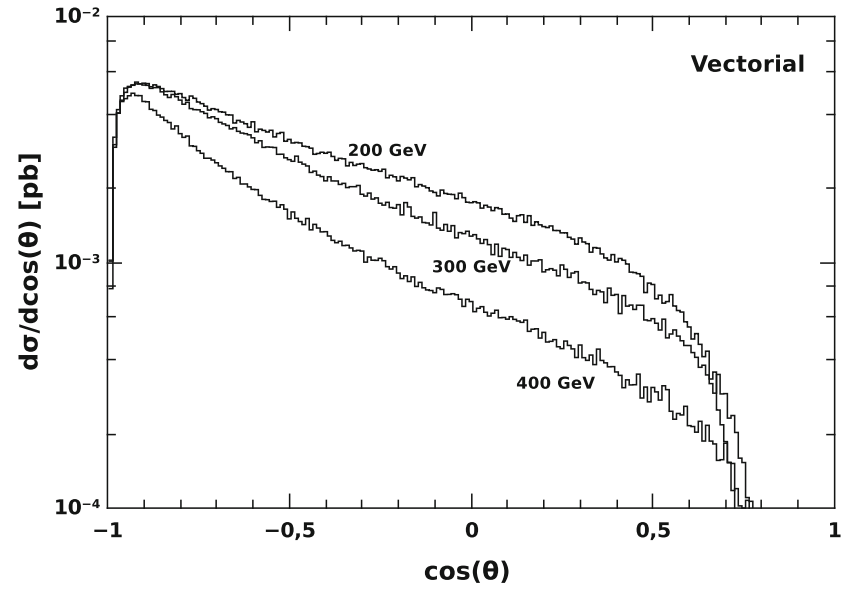

(a) Vectorial operators contribution. to each fermion family $i, j=1,2$ considered. Similarly, to study the contribution from the vectorial operators we set the couplings $\alpha_{S_{1,2,3}}^{(i, j)}$ equal to zero, and take $\alpha_{W}^{(i)}=\alpha_{V_{0}}^{(i, j)}$ equal to the values in Table 1.

In Fig. 5a, $\mathrm{b}$ we show the angular distribution for masses $m_{N}=200,300$ and $400 \mathrm{GeV}$ for the vectorial and scalar operators, respectively. The cuts presented in Fig. 4 are applied, and the beam energies considered are $E_{p}=7 \mathrm{TeV}$ and $E_{e}=150 \mathrm{GeV}$ through the following. We find an asymmetric distribution for both coupling sets, favoring the backward direction, as the outgoing anti-lepton is boosted in the proton beam direction. One can also see the scalar operators contribution to the cross section is about an order of magnitude less than the vectorial contribution.

In order to estimate the chances of disentangling the contributions from both operator sets, we study the angular asymmetry $A_{\mathrm{FB}}^{l^{+}}$, taking into account the error

$\Delta A_{\mathrm{FB}}^{l^{+}}=\sqrt{\left(\frac{\partial A_{\mathrm{FB}}^{l^{+}}}{\partial N_{+}}\right)^{2}\left(\Delta N_{+}\right)^{2}+\left(\frac{\partial A_{\mathrm{FB}}^{l^{+}}}{\partial N_{-}}\right)^{2}\left(\Delta N_{-}\right)^{2}}$.

Assuming the number of events to be Poisson distributed, we write

$\Delta N_{+}=\sqrt{N_{+}}$and $\Delta N_{-}=\sqrt{N_{-}}$

and a straightforward calculation leads to

$\Delta A_{\mathrm{FB}}^{l^{+}}=\sqrt{\frac{1-\left(A_{\mathrm{FB}}^{l^{+}}\right)^{2}}{N_{+}+N_{-}}}$.

The results for the $A_{\mathrm{FB}}^{l^{+}}$observable for the vectorial and scalar operators are shown in Fig. 6. Here we assume a baseline integrated luminosity of $\mathcal{L}=1 a b^{-1}$. We can see a clear sep-

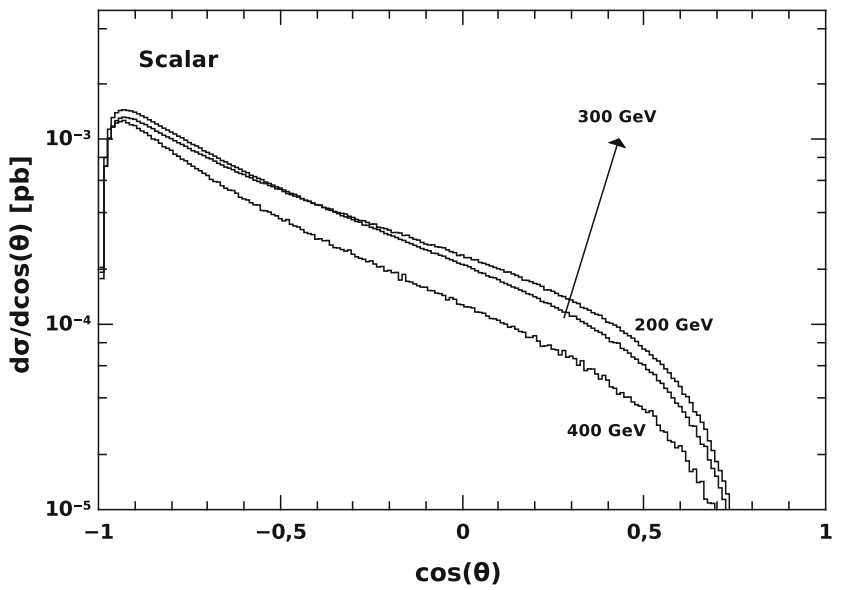

(b) Scalar operators contribution.

Fig. 5 Angular distribution for the contribution to the cross section of vectorial and scalar operators for different Majorana neutrino masses 


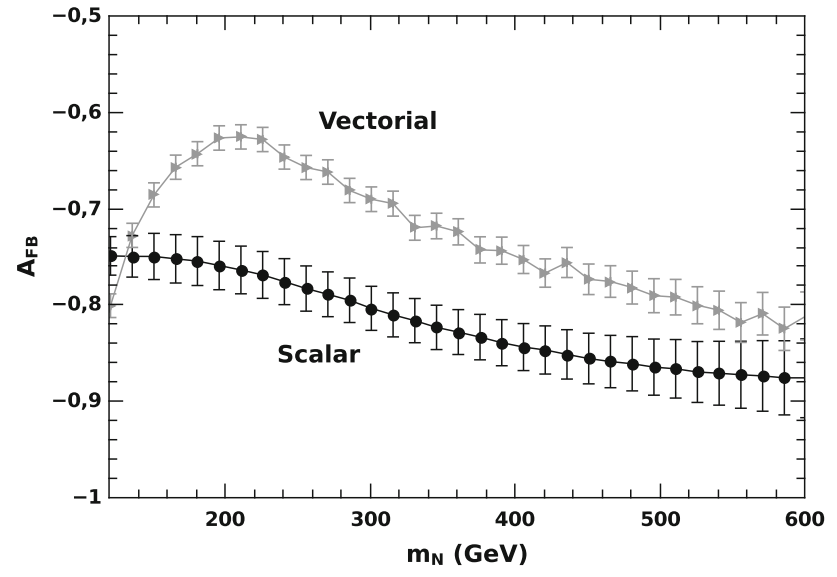

Fig. 6 Asymmetry in the angular distribution of the final anti-lepton, with the errors estimates as defined in the text

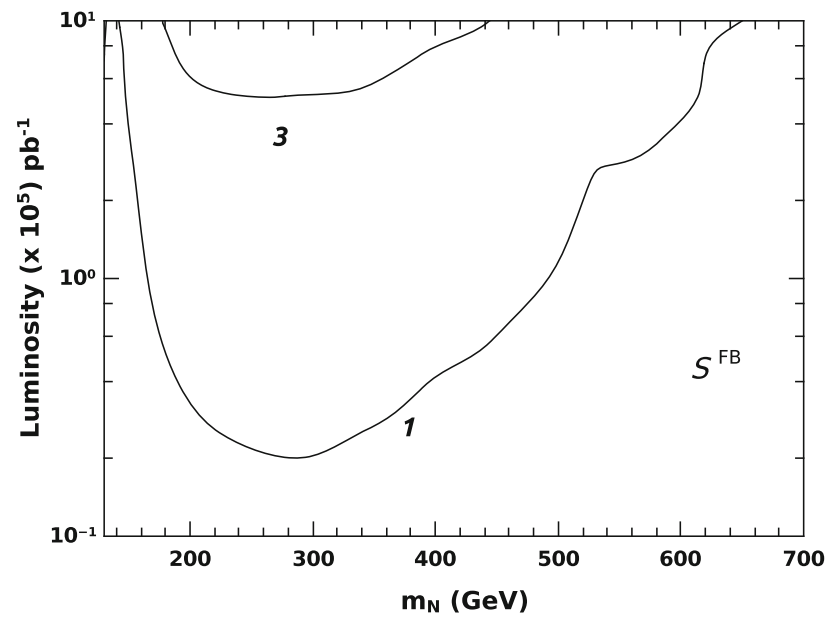

Fig. 7 Contour plot for the observable $\mathcal{S}^{\mathrm{FB}}$ as defined in the text

aration between the contributions of both sets, which could help distinguishing between different kinds of new physics driving the sterile neutrino interactions.

We can test the ability of the forward-backward asymmetry to separate the effects of vectorial and scalar operators defining the quantity $\mathcal{S}^{\mathrm{FB}}$ :

$\mathcal{S}^{\mathrm{FB}}=\frac{A_{\mathrm{FB}, \mathrm{vec}}^{l^{+}}-A_{\mathrm{FB}, \mathrm{sca}}^{l^{+}}}{\Delta A_{\mathrm{FB}, \mathrm{vec}}^{l^{+}}+\Delta A_{\mathrm{FB}, \mathrm{sca}}^{l^{+}}}$.

In Fig. 7 we show the contour plot for different values of $\mathcal{S}^{\mathrm{FB}}$ in the mass-luminosity $\left(m_{N}, \mathcal{L}\right)$ plane. The magnitude of $\mathcal{S}^{\mathrm{FB}}$ represents the number of standard deviations between the contributions to the asymmetry from the vectorial and scalar operators. We find that the $\mathrm{LHeC}$ could well disentangle both effects for Majorana neutrinos in a mass range $m_{N} \sim 200-400 \mathrm{GeV}$ within an operation year, when the luminosity reaches $1 \mathrm{ab}^{-1}$.

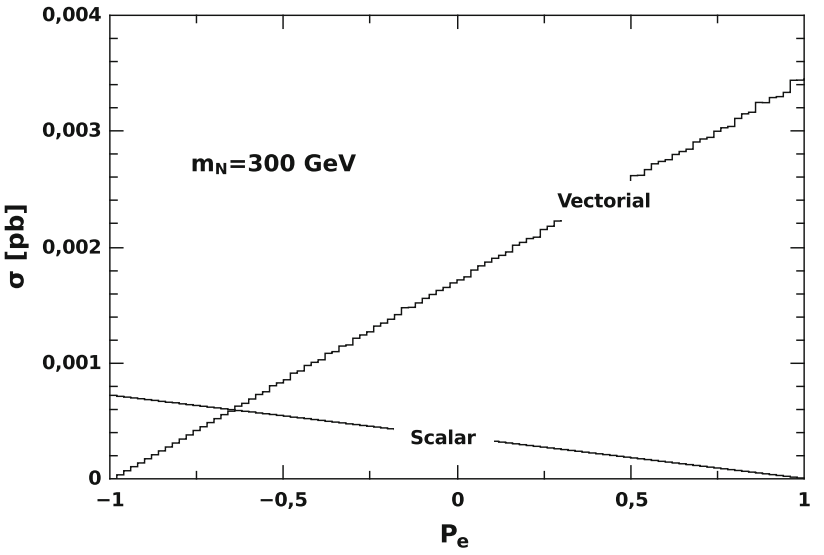

Fig. 8 Cross section dependence with $P_{e}$ for the vectorial and scalar operators

\section{Initial electron polarization}

The initial electron polarization $P_{e}$ can also be used to distinguish the vectorial and scalar operators contribution. It has been exploited recently in the case of the left-right symmetric model, where a right-polarized initial electron enhances the right handed charged current interaction [22,24].

The cross section for the process $e^{-} p \rightarrow l_{j}^{+}+3$ jets in (8) can be written in terms of $P_{e}$ as

$\hat{\sigma}_{i}=\frac{1}{2}\left(1+P_{e}\right) \hat{\sigma}_{i}^{R}+\frac{1}{2}\left(1-P_{e}\right) \hat{\sigma}_{i}^{L}$

where

$\hat{\sigma}_{i}^{R(L)}(x s)=\int(2 \pi)^{4} \delta^{(4)}\left(p_{e}+p_{u}-\sum_{j=1,4} k_{j}\right) \overline{\left|M_{(i)}^{R(L)}\right|^{2}} \prod_{j=1,4} \frac{d^{4} k_{j}}{2 \pi^{3}}$.

Thus, we can write again in the narrow width approximation:

$$
\begin{aligned}
{\overline{\mid M_{(i)}^{R(L)}}}^{2}= & \left(\frac{\pi}{4 m_{N} \Gamma_{N} \hat{s}}\right) \delta\left(k_{N}^{2}-m_{N}^{2}\right)\left|\Lambda_{(I), i}^{R(L)}\right|^{2}\left(\left|\Lambda_{(I I)}^{(+)}\right|^{2}\right. \\
& \left.+\left|\Lambda_{I I}^{(-)}\right|^{2}\right)
\end{aligned}
$$

where

$$
\begin{aligned}
\left|\Lambda_{(I), i}^{R}\right|^{2}= & \frac{16}{\Lambda^{2}}\left[\left(\alpha_{W}^{2}\left|\Pi_{W}^{(2)}\right|^{2}\right)\left(k_{d} \cdot p_{e}\right)\left(k_{N} \cdot p_{u}\right)\right. \\
& \left.+\left(\alpha_{V_{0}}^{2}\right)\left(k_{d} \cdot k_{N}\right)\left(p_{e} \cdot p_{u}\right)\right]
\end{aligned}
$$

and

$$
\begin{aligned}
\left|\Lambda_{(I), i}^{L}\right|^{2}= & \frac{4}{\Lambda^{2}}\left[4\left(\alpha_{S_{2}}\left(\alpha_{S_{2}}-\alpha_{S_{3}}\right)+\alpha_{S_{1}}^{2}\right)\left(k_{d} \cdot p_{u}\right)\left(k_{N} \cdot p_{e}\right)\right. \\
& +\alpha_{S_{3}}\left(\alpha_{S_{3}}-\alpha_{S_{2}}\right)\left(k_{d} \cdot p_{e}\right)\left(k_{N} \cdot p_{u}\right) \\
& \left.+\alpha_{S_{3}} \alpha_{S_{2}}\left(k_{d} \cdot k_{N}\right)\left(p_{e} \cdot p_{u}\right)\right] .
\end{aligned}
$$




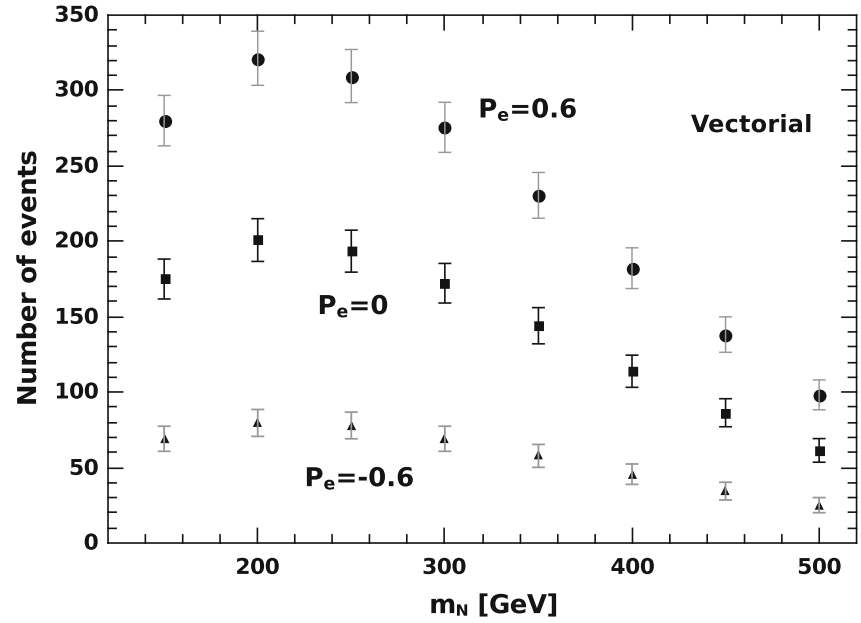

(a) Vectorial operators contribution.

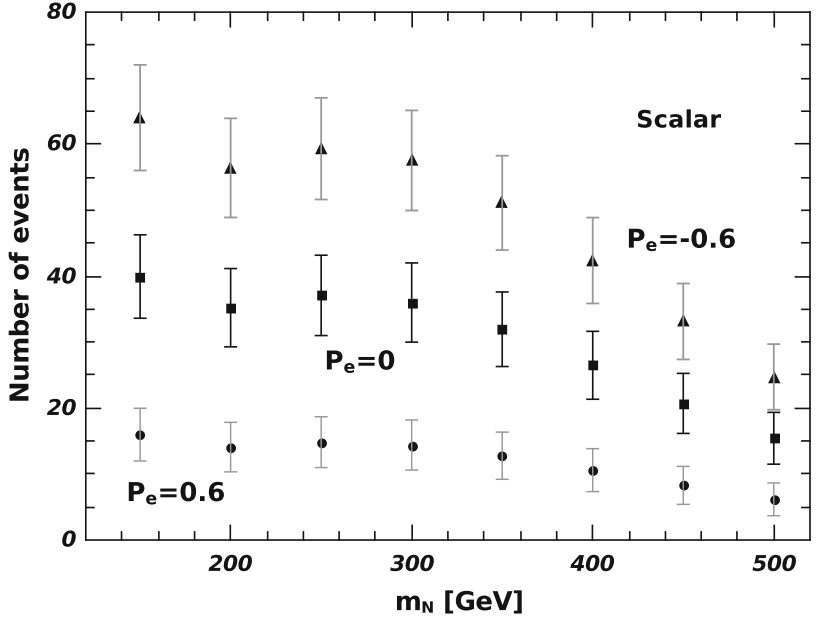

(b) Scalar operators contribution.

Fig. 9 Number of events and error bars for the signal with $P_{e}=-0.6,0$ and 0.6 . Here $\mathcal{L}=100 \mathrm{fb}^{-1}$. a Vectorial operators contribution. b Scalar operators contribution

From these expressions we can clearly see that the vectorial operators contribute to the right $(R)$ part, and the scalars to the left $(L)$ part of the cross section.

In Fig. 8 we show the behavior of the cross section with the polarization for $m_{N}=300 \mathrm{GeV}$ for the contribution of vectorial and scalar operators. In Fig. 9a, b we show the vectorial and scalar contributions respectively as a function of the Majorana neutrino mass for unpolarized $\left(P_{e}=0\right)$, left and right-polarized electrons $\left(P_{e}= \pm 0.6\right)$, for an integrated luminosity $\mathcal{L}=100 \mathrm{fb}^{-1}$.

Finally, in analogy with the last section we define the function $\mathcal{S}^{\text {pol }}$

$\mathcal{S}^{\mathrm{pol}}=\frac{N^{\mathrm{vec}}-N^{\mathrm{sca}}}{\sqrt{N^{\mathrm{vec}}}+\sqrt{N^{\mathrm{sca}}}}$

which represents the number of standard deviations between the numbers of events produced by the vectorial and the scalar operators contributions. We have considered the contour plot for new function $\mathcal{S}^{\text {pol }}$ in Fig. 10, again for $\mathcal{L}=100 \mathrm{fb}^{-1}$. Also here it is possible to see regions where the contributions of the different operators are considerably separated. Indeed, the polarization analysis is more promising than the angular asymmetry to distinguish the different operators contributions for a right-polarized electron beam.

\section{Summary and conclusions}

The heavy neutrino effective field theory parameterizes highscale weakly coupled physics beyond the minimal seesaw mechanism in a model independent framework, allowing for sizable lepton number violating (LNV) effects in colliders. While models like the minimal seesaw mechanism lead to

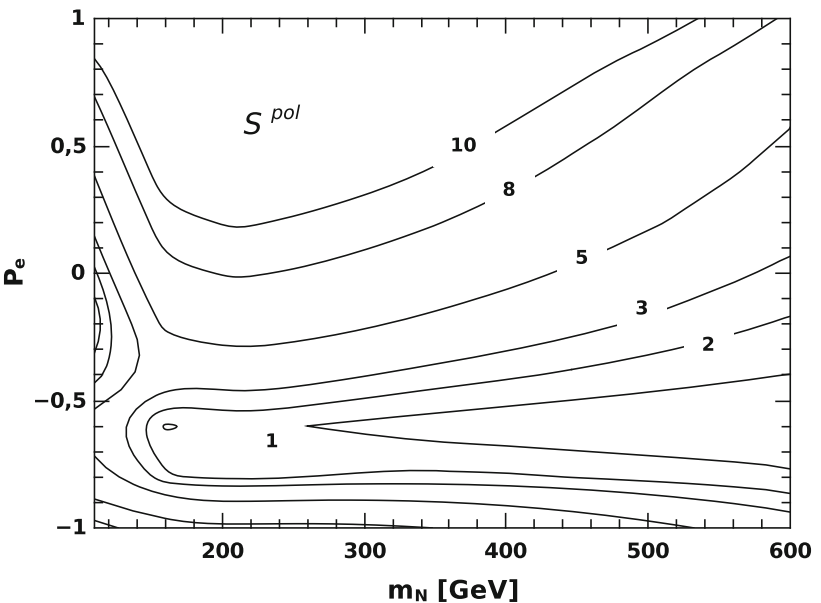

Fig. 10 Contour plot for different values of the function $\mathcal{S}^{\text {pol }}$, for $\mathcal{L}=$ $100 \mathrm{fb}^{-1}$

the decoupling of the heavy Majorana neutrinos, predicting unobservable LNV, the effective Lagrangian framework considered in this work could serve as a means to discern between the different possible kinds of effective interactions contributing to the $e^{-} p \rightarrow l_{j}^{+}+3$ jets signal at the LHeC. In particular, we studied the capability of an angular observable and the initial electron polarization to disentangle the contributions of vectorial and scalar dimension- 6 effective operators.

In this paper we have calculated the total unpolarized cross section $\sigma\left(e^{-} p \rightarrow l_{j}^{+}+3\right.$ jets $)$ in the LHeC for different values of $m_{N}$ for proton and electron beams of $E_{p}=7 \mathrm{TeV}$ and $E_{e}=150 \mathrm{GeV}$, respectively, updating the numerical values of the effective couplings $\alpha_{\mathcal{J}}$ to the existing experimental bounds, and implemented cuts in the phase space that can help to enhance the signal to background relation. 
In order to discern the contribution of the different operators we calculate a forward-backward angular asymmetry for the final anti-lepton. We present our results for the asymmetry as a function of the heavy neutrino mass, for the vectorial and scalar effective interactions, considering the contour plot for the observable $\mathcal{S}^{\mathrm{FB}}$, which measures the distance in standard deviations between the contributions to the asymmetry from the vectorial and scalar operators as a function of the heavy neutrino mass and the integrated luminosity.

We calculate the effect on the signal number of events for the vectorial and scalar interactions when the initial electron is polarized. We present the results varying the Majorana neutrino mass and the electron polarization, taking the number of events to be Poisson distributed and considering the corresponding errors. Finally, we have studied the contour plot for the new function $\mathcal{S}^{\text {pol }}$ defined in analogy with $\mathcal{S}^{\mathrm{FB}}$. Also in the polarization analysis it is possible to see regions where the contributions of the different operators are considerably separated.

Our findings show that electron-proton colliders, being complementary facilities to both $e^{+} e^{-}$and $p p$ machines which provide higher center-of-mass energies than the former and a cleaner environment than the latter, allow for detailed angular and polarization studies and could improve our knowledge on possible Majorana neutrinos and their interactions, which is a fundamental unsolved puzzle in particle physics.

Acknowledgements We thank CONICET (Argentina) and Universidad Nacional de Mar del Plata (Argentina); and PEDECIBA and CSICUdelaR (Uruguay) for their financial supports.

Open Access This article is distributed under the terms of the Creative Commons Attribution 4.0 International License (http://creativecomm ons.org/licenses/by/4.0/), which permits unrestricted use, distribution, and reproduction in any medium, provided you give appropriate credit to the original author(s) and the source, provide a link to the Creative Commons license, and indicate if changes were made.

Funded by SCOAP ${ }^{3}$.

\section{References}

1. P. Minkowski, $\mu \rightarrow e \gamma$ at a rate of one out of 1-billion muon decays? Phys. Lett. B67, 421 (1977)

2. R.N. Mohapatra, G. Senjanovic, Neutrino mass and spontaneous parity violation. Phys. Rev. Lett. 44, 912 (1980)

3. T. Yanagida, Horizontal symmetry and masses of neutrinos. Prog. Theor. Phys. 64, 1103 (1980)

4. M. Gell-Mann, P. Ramond, R. Slansky, Complex spinors and unified theories. Conf. Proc. C790927, 315 (1979). arXiv:1306.4669 [hep-th]

5. J. Schechter, J.W.F. Valle, Neutrino masses in $\mathrm{SU}(2) \times \mathrm{U}(1)$ theories. Phys. Rev. D22, 2227 (1980)

6. B. Kayser, F. Gibrat-Debu, F. Perrier, The physics of massive neutrinos. World Sci. Lect. Notes Phys. 25, 1 (1989)

7. C. Patrignani et al. (Particle Data Group), Review of particle physics. Chin. Phys. C 40, 100001 (2016)
8. Y. Cai, T. Han, T. Li, R. Ruiz, Lepton-number violation: seesaw models and their collider tests (2017). arXiv:1711.02180 [hep-ph]

9. A. Atre, T. Han, S. Pascoli, B. Zhang, The search for heavy Majorana neutrinos. JHEP 0905, 030 (2009). arXiv:0901.3589 [hep-ph]

10. F. del Aguila, S. Bar-Shalom, A. Soni, J. Wudka, Heavy Majorana neutrinos in the effective lagrangian description: application to hadron colliders. Phys. Lett. B 670, 399 (2009). arXiv:0806.0876 [hep-ph]

11. L. Duarte, O.A. Sampayo, in Physical and Mathematical Aspects of Symmetries. ed. by S. Duarte, J.-P. Gazeau, S. Faci, T. Micklitz, R. Scherer, F. Toppan (Springer International Publishing, Cham, 2017), pp. 153-158 (ISBN 978-3-319-69164-0)

12. F. del Aguila, J.A. Aguilar-Saavedra, R. Pittau, Heavy neutrino signals at large hadron colliders. JHEP 10, 047 (2007). arXiv:hep-ph/0703261 [hep-ph]

13. F.F. Deppisch, P.S. Bhupal Dev, A. Pilaftsis, Neutrinos and collider physics. N. J. Phys. 17, 075019 (2015). arXiv:1502.06541 [hep-ph]

14. S. Antusch, E. Cazzato, O. Fischer, Sterile neutrino searches at future $e^{-} e^{+}, p p$, and $e^{-} p$ colliders. Int. J. Mod. Phys. A32, 1750078 (2017). arXiv:1612.02728 [hep-ph]

15. C. Blaksley, M. Blennow, F. Bonnet, P. Coloma, E. FernandezMartinez, Heavy neutrinos and lepton number violation in lp colliders. Nucl. Phys. B852, 353 (2011). arXiv:1105.0308 [hep-ph]

16. H. Liang, X.-G. He, W.-G. Ma, S.-M. Wang, R.-Y. Zhang, Seesaw type I and III at the LHeC. JHEP 1009, 023 (2010). arXiv:1006.5534 [hep-ph]

17. G. Ingelman, J. Rathsman, Heavy Majorana neutrinos at e p colliders. Z. Phys. C60, 243 (1993)

18. W. Buchmuller, C. Greub, Heavy Majorana neutrinos in electronpositron and electron-proton collisions. Nucl. Phys. B363, 345 (1991)

19. O. Bruening, M. Klein, The Large Hadron Electron Collider. Mod. Phys. Lett. A 28, 1330011 (2013). arXiv:1305.2090 [physics.acc$\mathrm{ph}]$

20. J. Abelleira Fernandez, et al. (LHeC Study Group), A large Hadron electron collider at CERN: report on the Physics and Design Concepts for Machine and Detector. J. Phys. G 39, 075001 (2012). arXiv:1206.2913 [physics.acc-ph]

21. D. Curtin, K. Deshpande, O. Fischer, J. Zurita, New physics opportunities for long-lived particles at electron-proton colliders (2017). arXiv:1712.07135 [hep-ph]

22. S. Mondal, S.K. Rai, Polarized window for left-right symmetry and a right-handed neutrino at the large Hadron-Electron collider. Phys. Rev. D93, 011702 (2016). arXiv:1510.08632 [hep-ph]

23. S. Mondal, S.K. Rai, Probing the heavy neutrinos of inverse seesaw model at the LHeC. Phys. Rev. D94, 033008 (2016). arXiv:1605.04508 [hep-ph]

24. M. Lindner, F.S. Queiroz, W. Rodejohann, C.E. Yaguna, Left-right symmetry and lepton number violation at the large Hadron Electron collider. JHEP 06, 140 (2016). arXiv:1604.08596 [hep-ph]

25. A. Aparici, K. Kim, A. Santamaria, J. Wudka, Right-handed neutrino magnetic moments. Phys. Rev. D80, 013010 (2009). arXiv:0904.3244 [hep-ph]

26. P. Ballett, S. Pascoli, M. Ross-Lonergan, MeV-scale sterile neutrino decays at the fermilab short-baseline neutrino program. JHEP 04, 102 (2017). arXiv: 1610.08512 [hep-ph]

27. A. Caputo, P. Hernandez, J. Lopez-Pavon, J. Salvado, The seesaw portal in testable models of neutrino masses. JHEP 06, 112 (2017). arXiv:1704.08721 [hep-ph]

28. S. Bhattacharya, J. Wudka, Dimension-seven operators in the standard model with right handed neutrinos. Phys. Rev. D 94, 055022 (2016). arXiv:1505.05264 [hep-ph] [Erratum: Phys. Rev. D 95(3), 039904 (2017)]

29. Y. Liao, X.-D. Ma, Operators up to dimension seven in standard model effective field theory extended with sterile neutrinos. Phys. Rev. D96, 015012 (2017). arXiv:1612.04527 [hep-ph] 
30. J. Peressutti, I. Romero, O.A. Sampayo, Majorana neutrinos production at NLC in an effective approach. Phys. Rev. D84, 113002 (2011). arXiv:1110.0959 [hep-ph]

31. J. Peressutti, O.A. Sampayo, Majorana neutrinos in $e \gamma$ colliders from an effective Lagrangian approach. Phys. Rev. D90, 013003 (2014)

32. L. Duarte, G.A. González-Sprinberg, O.A. Sampayo, Majorana neutrinos production at $\mathrm{LHeC}$ in an effective approach. Phys. Rev. D91, 053007 (2015). arXiv:1412.1433 [hep-ph]

33. L. Duarte, J. Peressutti, O.A. Sampayo, Majorana neutrino decay in an effective approach. Phys. Rev. D92, 093002 (2015). arXiv:1508.01588 [hep-ph]

34. L. Duarte, I. Romero, J. Peressutti, O.A. Sampayo, Effective Majorana neutrino decay. Eur. Phys. J. C76, 453 (2016). arXiv:1603.08052 [hep-ph]

35. L. Duarte, J. Peressutti, O.A. Sampayo, Not-that-heavy Majorana neutrino signals at the LHC. J. Phys. G45, 025001 (2018). arXiv:1610.03894 [hep-ph]

36. C.-X. Yue, Y.-C. Guo, Z.-H. Zhao, Majorana neutrino signals at Belle-II and ILC. Nucl. Phys. B925, 186 (2017). arXiv:1710.06144 [hep-ph]

37. J. Wudka, A short course in effective Lagrangians. AIP Conf. Proc. 531, 81 (2000). arXiv:hep-ph/0002180

38. S. Weinberg, Baryon and lepton nonconserving processes. Phys. Rev. Lett. 43, 1566 (1979)

39. C. Arzt, M. Einhorn, J. Wudka, Patterns of deviation from the standard model. Nucl. Phys. B433, 41 (1995). arXiv:hep-ph/9405214

40. R. Ruiz, Lepton number violation at colliders from kinematically inaccessible gauge bosons. Eur. Phys. J. C77, 375 (2017). arXiv:1703.04669 [hep-ph]

41. A. de Gouvêa, A. Kobach, Global constraints on a heavy neutrino. Phys. Rev. D93, 033005 (2016). arXiv:1511.00683 [hep-ph]

42. S. Antusch, O. Fischer, Testing sterile neutrino extensions of the standard model at future lepton colliders. JHEP 05, 053 (2015). arXiv:1502.05915 [hep-ph]
43. A. Das, P.S. Bhupal Dev, N. Okada, Direct bounds on electroweak scale pseudo-Dirac neutrinos from $\sqrt{s}=8 \mathrm{TeV}$ LHC data. Phys. Lett. B735, 364 (2014). arXiv:1405.0177 [hep-ph]

44. E. Fernandez-Martinez, J. Hernandez-Garcia, J. Lopez-Pavon, Global constraints on heavy neutrino mixing. JHEP 08, 033 (2016). arXiv:1605.08774 [hep-ph]

45. A. Gando et al. (KamLAND-Zen), Search for Majorana neutrinos near the inverted mass hierarchy region with KamLAND-Zen. Phys. Rev. Lett. 117, 082503 (2016). arXiv:1605.02889 [hep-ex] [Addendum: Phys. Rev. Lett. 117(10), 109903 (2016)]

46. R. Mohapatra, Particle physics implications of neutrinoless double beta decay. Nucl. Phys. Proc. Suppl. 77, 376 (1999). arXiv:hep-ph/9808284

47. D. Tommasini, G. Barenboim, J. Bernabeu, C. Jarlskog, Nondecoupling of heavy neutrinos and lepton flavor violation. Nucl. Phys. B444, 451 (1995). arXiv:hep-ph/9503228

48. F. Aguila, J. de Blas, M. Perez-Victoria, Effects of new leptons in Electroweak Precision Data. Phys. Rev. D 78, 013010 (2008). arXiv:0803.4008 [hep-ph]

49. J. Pumplin, D.R. Stump, J. Huston, H.L. Lai, P.M. Nadolsky, W.K. Tung, New generation of parton distributions with uncertainties from global QCD analysis. JHEP 07, 012 (2002). arXiv:hep-ph/0201195

50. R. Kleiss, W.J. Stirling, S. Ellis, A new Monte Carlo treatment of multiparticle phase space at high-energies. Comput. Phys. Commun. 40, 359 (1986)

51. A. Belyaev, N.D. Christensen, A. Pukhov, CalcHEP 3.4 for collider physics within and beyond the standard model. Comput. Phys. Commun. 184, 1729 (2013). arXiv:1207.6082 [hep-ph]

52. C. Arbelaéz, C. Dib, I. Schmidt, J.C. Vasquez, Probing the dirac or Majorana nature of the heavy neutrinos in pure leptonic decays at the LHC. Phys. Rev. D97, 055011 (2018). arXiv:1712.08704 [hep-ph] 